\title{
On high proper motion white dwarfs from photographic surveys
}

\author{
C. Reylé1, A. C. Robin ${ }^{1}$, and M. Crézé2 \\ 1 CNRS UMR6091, Observatoire de Besançon, BP1615, 25010 Besançon Cedex, France \\ 2 Université de Bretagne-Sud, BP 573, 56017 Vannes Cedex, France
}

Received 13 August 2001 / Accepted 15 September 2001

\begin{abstract}
The interpretation of high proper motion white dwarfs detected by Oppenheimer et al. (2001) was the start of a lively controversy. While the discoverers identify a large fraction of their findings as dark halo members, others interpret the same sample as essentially made of disc and/or thick disc stars. We use the comprehensive description of Galactic stellar populations provided by the "Besançon" model to produce a realistic simulation of Oppenheimer et al. data, including all observational selections and calibration biases. The conclusion is unambiguous: Thick disc white dwarfs resulting from ordinary hypotheses on the local density and kinematics are sufficient to explain the observed objects, there is no need for halo white dwarfs. This conclusion is robust to reasonable changes in model ingredients. The main cause of the misinterpretation seems to be that the velocity distribution of a proper motion selected star sample is severely biased in favour of high velocities. This has been neglected in previous analyses. Obviously this does not prove that no such objects like halo white dwarfs can exist, but Oppenheimer et al. observations drive their possible contribution in the dark matter halo down to an extremely low fraction.
\end{abstract}

Key words. cosmology: dark matter - Galaxy: structure - Galaxy: stellar content - Galaxy: general

\section{Introduction}

In a recent paper, Oppenheimer et al. (2001) (hereafter OHDHS) give evidence for a large number of faint white dwarfs detected in digitized photographic plates from the SuperCOSMOS Sky Survey ${ }^{1}$. Their interpretation of these stars as dark halo members was the start of a controversy. While Reid et al. (2001) and more recently Graff (2001) interpret OHDHS stars as the high velocity tail of the disc/thick disc populations, Koopmans \& Blandford (2001) argue that there is a statistically significant break in the velocity distribution, which they interpret as the thick disc/halo break. They propose a dynamical mechanism to produce these "halo" objects out of the disc. Another line of arguments has been developed by Gibson \& Flynn (2001) who point out a number of biases in OHDHS analysis all adding up to produce an overestimation of the local density of halo white dwarfs, but the authors do not discuss their nature. In addition, Hansen (2001) finds a similar age distribution for this population as those in the thin disc white dwarf population. In order to clarify the situation, we use the Besançon model of stellar populations in the Milky Way to simulate OHDHS observations. In the next section we describe model

Send offprint requests to: C. Reylé,

e-mail: celine@obs-besancon.fr

1 See http://www-wfau.roe.ac.uk/sss hypothesis and the construction of the simulated sample, the third section being dedicated to the discussion and conclusions.

\section{Simulations}

We have performed simulations from the Besançon Galactic model using the following assessment: disc stars are assumed to have ages from 0 to $10 \mathrm{Gyr}$, with appropriate kinematics as a function of age (Haywood et al. 1997). The density laws are Einasto discs, which are close to $\operatorname{sech}^{2}$ vertically (Bienaymé et al. 1987a,b). The white dwarf luminosity function is from Sion \& Liebert (1977) and the photometry is derived from Bergeron et al. (1995) model atmospheres. The ellipticities of these components are a function of age, and constrained by the Galactic potential through the Boltzmann equation. The average kinematics of white dwarfs in a volume limited sample in the solar neighbourhood result in a velocity ellipsoid (42.1, 27.2, 17.2) and an asymmetric drift of $16.6 \mathrm{~km} \mathrm{~s}^{-1}$.

The thick disc population is modeled as originating from a single epoch of star formation. The adopted thick disc density law is described in Reylé \& Robin (2001), as fitted to large sets of available star counts. The local density is $10^{-3} \mathrm{pc}^{-3}$ or $7.1 \times 10^{-4} M_{\odot} \mathrm{pc}^{-3}\left(M_{V}<8\right)$, that is about $6 \%$ of the disc local density. In order to ensure continuity of the density gradient at $z=0$, the 
density law is made quadratic near the plane $(z<337 \mathrm{pc})$. Further out from the plane, the density law follows an exponential of scale height $850 \mathrm{pc}$.

The white dwarf luminosity function (hereafter WDLF) of the thick disc has been computed by GarciaBerro (private communication) from the model of Isern et al. (1998) assuming a Salpeter initial mass function (IMF) and an age of 12 Gyr. We attempted to normalise the WDLF by computing the number of white dwarfs relative to main sequence stars, assuming that all stars with a mass greater than the mass at the turnoff $\left(M_{\mathrm{TO}}=0.83 M_{\odot}\right)$ are now white dwarfs. We consider a two slope IMF, the lower mass parts being constrained by available star counts (Reylé \& Robin 2001) accounting for a binary correction:

$$
\begin{array}{ll}
\mathrm{d} N / \mathrm{d} m \propto m^{-2.35} ; & M_{\mathrm{TO}}<m<8 M_{\odot} \\
\mathrm{d} N / \mathrm{d} m \propto m^{-0.75} ; & 0.1 M_{\odot}<m<M_{\mathrm{TO}} .
\end{array}
$$

The ratio of white dwarfs to main sequence stars is therefore:

$$
\frac{N_{\mathrm{WD}}}{N_{\mathrm{SP}}}=\frac{0.74 \int_{M_{\mathrm{TO}}}^{8 M_{\odot}} m^{-2.35} \mathrm{~d} m}{\int_{0.1 M_{\odot}}^{M_{\mathrm{TO}}} m^{-0.75} \mathrm{~d} m}=0.43 .
$$

The factor 0.74 ensures the continuity at the turnoff. However, it appears that the predicted number of thick disc white dwarfs, with these assumptions, is much higher than the number of observed white dwarfs in the OHDHS sample. Several reasons could be invoked: first, we do not know how the OHDHS sample is complete, specially near the limiting magnitude. Second, hypotheses which have led to the estimation of the thick disc WDLF have not been tested yet because these stars are not identified as such in current white dwarfs samples: Uncertainties remain on the IMF slope (at high mass, with no observational constraints, we have chosen the conservative Salpeter IMF, while at low mass an IMF slope $\alpha=1$, still within our error bars, drives down the fraction to 0.33 ). Uncertainties remain also on the star formation history, which may be more complex than a single burst. These reasons led us to normalize the thick disc WDLF in order to be in agreement with the OHDHS sample: the fraction of white dwarfs over sub-turnoff stars drops down to $20 \%$, about the value of Hansen (2001), corresponding to a local density of $5 \times 10^{-4}$ stars $\mathrm{pc}^{-3}$. This normalisation is also in agreement with the number of white dwarfs in the expanded LHS (Luyten Half Second proper motion catalogue) white dwarf sample (Liebert et al. 1999). The velocity ellipsoid of the thick disc is constrained by photoastrometric survey in several Galactic directions (Ojha et al. 1996$)$ to be $(67,51,42)$ while the asymmetric drift is $53 \mathrm{~km} \mathrm{~s}^{-1}$.

Dark halo white dwarfs are modeled assuming that the dark halo is filled with ancient white dwarfs with a certain factor $f$. The dark halo local density is $8 \times$ $10^{-3} M_{\odot} \mathrm{pc}^{-3}$ from dynamical constraints (Bienaymé et al. 1987a). Hence the local density of halo white dwarfs is assumed to be $f$ times this value. As a first guess compatible with microlensing constraints, we set $f=0.1$ in this simulation. We use Bergeron et al. (1995) model atmospheres for hydrogen white dwarfs of mass $0.6 M_{\odot}$ to the turnover $\left(M_{V}<17\right)$, and Chabrier et al. (2000) models for the cooler part of the sequence. The halo white dwarfs luminosity function has been computed by Isern et al. (1998) with the ad hoc Chabrier (1999) IMF and an age of 12 or 14 Gyr. The space velocities of the dark halo are computed assuming the same kinematics as for the spheroid: a velocity ellipsoid $(131,106,85)$ and a null rotation, the LSR rotational velocity being $229 \mathrm{~km} \mathrm{~s}^{-1}$ (Bienaymé et al. 1987a).

The colours are computed by transforming the standard Johnson-Cousins system to the observed one using the equations given in the SuperCOSMOS Sky Survey description:

$$
\begin{aligned}
B_{\mathrm{J}}=B & -0.28 \times(B-V) ; \text { for }-0.1<B-V<1.6 \\
R 59 F= & R-0.006-0.059 \times(R-I)+0.112 \\
& \times(R-I)^{2}+0.0238 \times(R-I)^{3} .
\end{aligned}
$$

The selection of the simulated sample has been done strictly following the OHDHS process. We simulate a field of $4165 \mathrm{deg}^{2}$ at the south Galactic cap. We select a sample of stars with $R 59 F \leq 19.45$, and proper motions in the range 0.33 to $3^{\prime \prime} \mathrm{yr}^{-1}$, assuming a mean error of $0.04^{\prime \prime} \mathrm{yr}^{-1}$. In order to reproduce the space velocities deduced by OHDHS, from the resulting sample, we compute the reduced proper motions, absolute magnitudes $M_{B_{\mathrm{J}}}$, distances and space velocities $U$ and $V$ using their formulae.

\section{Results and discussion}

Figure 1a reproduces the observed distribution of the OHDHS sample in the $(U, V)$ plane. The ellipses indicate the $1 \sigma$ and $2 \sigma$ velocity dispersions as defined by OHDHS for the old disc (right) and the halo (left). Objects out of the $2 \sigma$ old disc ellipse are interpreted as dark halo white dwarfs by OHDHS (squares with boxes). This figure can be compared with the simulated distribution of the selected white dwarfs represented in Fig. 1b. The disc is coded with crosses, the thick disc with circles, and the dark halo with asterisks. Ellipses are the same as in Fig. 1a for comparison.

The vast majority of OHDHS white dwarfs out of the $2 \sigma$ old disc ellipse can in fact be interpreted as thick disc white dwarfs, as expected from ordinary hypotheses on the local density and kinematics of the thick disc white dwarfs, although it is not possible to be sure that none of these white dwarfs belong to the dark halo. Hansen (2001) argues that the OHDHS white dwarfs are not old enough to account for the thick disc population, given that most of the white dwarfs in the thick disc should be fainter in the case of a single burst of star formation in the thick disc 12 Gyr ago. However, the simulation shows that, even in the burst case, the number of younger white dwarfs in the 

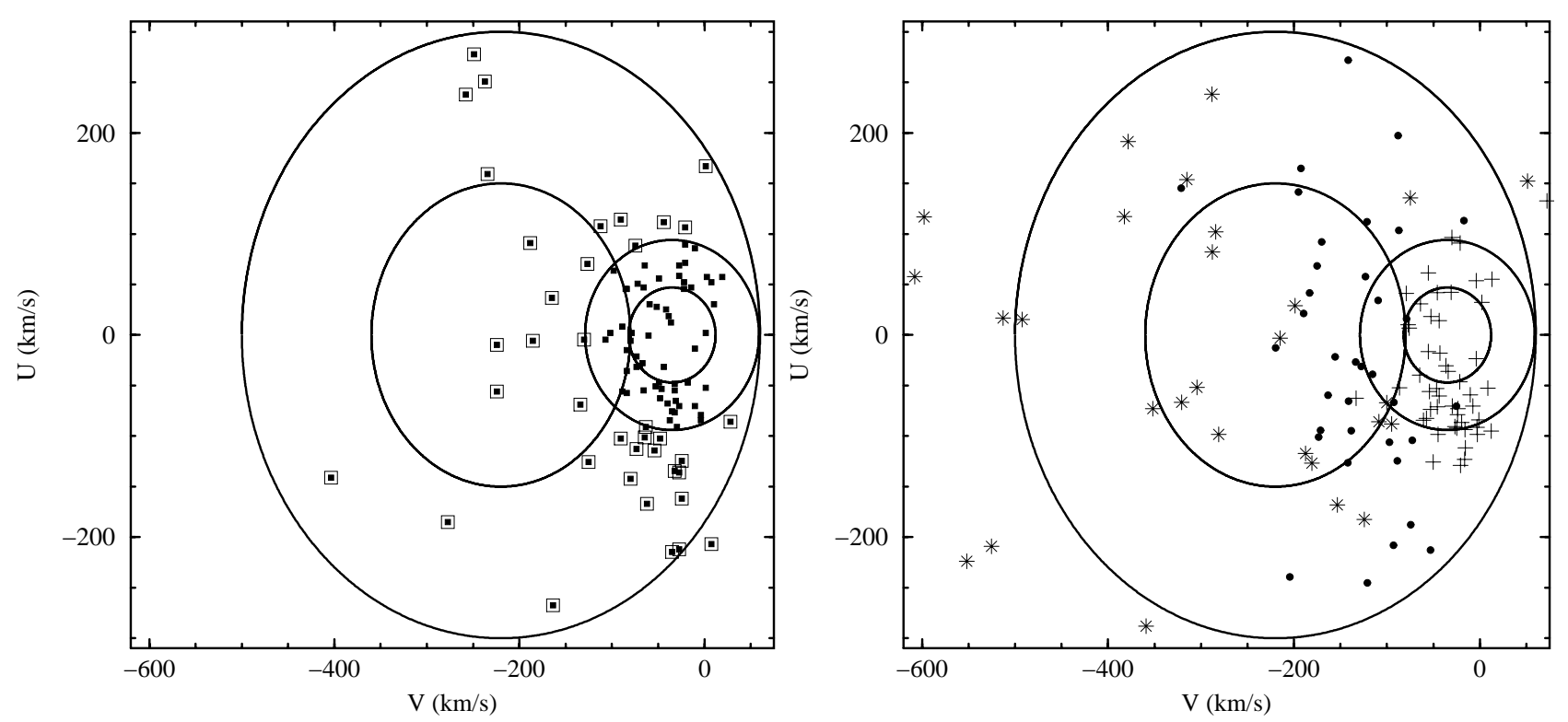

Fig. 1. Velocity space distribution of white dwarfs in the Oppenheimer et al. (2001) sample: a) Original observations. Objects with boxes are interpreted as halo stars. b) Simulation with a white dwarf local density being $10 \%(f=0.1)$ of the dark halo local density, and an age of 12 Gyr. Crosses: disc stars, circles: thick disc stars, asterisks: dark halo stars.

thick disc remains large enough to explain the OHDHS sample. They come from stars for which the time spent on the main sequence is not negligible. Actually, very few of the simulated stars are beyond the turnover in the white dwarf colour-magnitude diagram.

Another difference that appears in Fig. 1 is the number of high velocity stars: Considering an age of 12 Gyr for the halo white dwarfs and a fraction $f=0.1,10$ objects are expected to have $V<-400 \mathrm{~km} \mathrm{~s}^{-1}$ (9 simulations were performed to compute a mean value for this number of high velocity objects). None of the OHDHS white dwarfs are present in this part of the diagram, indicating that the halo white dwarf local density is no more than $1 \%$ of the dark halo local density, in agreement with the values derived by Koopmans \& Blandford (2001) and Gibson \& Flynn (2001). However, this upper-limit remains uncertain as the completeness of the OHDHS sample is not precisely determined. Furthermore, if considering an halo age of $14 \mathrm{Gyr}$, the expected number is 2 .

The photometric estimation of the distances could introduce a bias. They are determined from a linear colourmagnitude relation, with uncertainties around $20 \%$. The linear relation is no longer valid for old white dwarfs beyond the turnover in the colour-magnitude diagram. Using this relation leads to an overestimation of their luminosities and consequently, their distances and $V$ velocities. However, very few stars on the OHDHS sample are affected by this bias (see Fig. 1 of Koopmans \& Blandford 2001). On the contrary, the simulated sample considering a 12 Gyr dark halo with $f=0.1$ contains some of these old white dwarfs, which are the stars one is looking for to explain the dark matter halo.

Another strong bias comes from the selection of high proper motion stars which isolates a high velocity tail of each population. The ellipses in Fig. 1 characterize kinematics of a complete halo/thick disc sample, but are incorrect for a high proper motion sample. The simulated sample can be separated in three distinct populations and it well reproduces the observed dispersions and rotation lag. Stars within the $2 \sigma$ old disc ellipse are mainly disc stars, in contradiction with Koopmans \& Blandford (2001) who neglected this population. Out of the $2 \sigma$ old disc ellipse, stars are dominated by thick disc stars and a few disc stars. The fraction $f=0.1$ for an halo of 12 Gyr clearly overestimates the number of observed stars in the $2 \sigma$ halo ellipse and even more outside.

As shown in Table 1, due to the observational biases in the sample, the mean asymmetric drift of the resulting sample is much larger that the normal value for a complete sample in the solar neighbourhood. The expected values for halo white dwarfs are also given in Table 1 . There are much larger than the values found by OHDHS, implying that the halo population cannot dominate the sample.

It should also be noted that, even if a large uncertainty remains on the true local density of thick disc white dwarfs, our conclusions are robust to changes of hypothesis within this uncertainty, since they are mainly based on the velocity characteristics of the sample. Uncertainties on the thick disc kinematics also exist. Adopting a larger asymmetric drift, such as $89 \mathrm{~km} \mathrm{~s}^{-1}$ (as in Robin \& Chen 1993), would slightly displace the thick disc white dwarfs towards negative $V$, but our conclusions remain unchanged. Had we adopted an asymmetric drift small enough to displace most of the thick disc stars inside the $2 \sigma$ old disc ellipse, the fraction $f=0.1$ would underestimate the number of stars with $-400 \mathrm{~km} \mathrm{~s}^{-1}<V<-200 \mathrm{~km} \mathrm{~s}^{-1}$, but a higher value for $f$ would dramatically overestimates the number of high velocity stars with $V<-400 \mathrm{~km} \mathrm{~s}^{-1}$. 
Table 1. Rotational lag and velocity dispersion $\sigma_{U}$ for disc, thick disc and halo white dwarfs. Values in a complete sample are those in the original model. Values from the selected sample come from the simulation with the high proper motion and apparent magnitude selections. In the selected sample the computed velocity dispersion and lag are about twice what it would be in a complete sample.

\begin{tabular}{cccc}
\hline & & complete sample & selected sample \\
\hline \multirow{4}{*}{ lag } & disc & $16.6 \mathrm{~km} \mathrm{~s}^{-1}$ & $30 \mathrm{~km} \mathrm{~s}^{-1}$ \\
& thick disc & $53 \mathrm{~km} \mathrm{~s}^{-1}$ & $122 \mathrm{~km} \mathrm{~s}^{-1}$ \\
& halo & $229 \mathrm{~km} \mathrm{~s}^{-1}$ & $336 \mathrm{~km} \mathrm{~s}^{-1}$ \\
\hline \multirow{4}{*}{$\sigma_{U}$} & disc & $42.1 \mathrm{~km} \mathrm{~s}^{-1}$ & $64 \mathrm{~km} \mathrm{~s}^{-1}$ \\
& thick disc & $67 \mathrm{~km} \mathrm{~s}^{-1}$ & $112 \mathrm{~km} \mathrm{~s}^{-1}$ \\
& halo & $131 \mathrm{~km} \mathrm{~s}^{-1}$ & $207 \mathrm{~km} \mathrm{~s}^{-1}$ \\
\hline
\end{tabular}

\section{Conclusions}

The simulated sample, taking into account all observational bias, gives an unambiguous result: most high velocity white dwarfs in the OHDHS sample can be safely interpreted as disc and thick disc stars. The expected contribution of spheroid white dwarfs, assuming their mass density to be $1.3 \times 10^{-5} M_{\odot} \mathrm{pc}^{-3}$ Gould et al. (1998) would be 0.5 stars. The OHDHS sample has been misinterpreted because the bias introduced by the selection of high proper motions has been neglected. Although some of the stars in the sample may be part of the halo, it is not necessary to call for exotic objects such as white dwarfs in the dark halo. However, this sample provides a direct observation of thick disc white dwarfs. Such observations will lead to the construction of the luminosity function for thick disc white dwarfs, and hence to a better understanding of the Milky Way's history.

The simulation also shows that a sample dominated by the dark halo white dwarfs would have much larger velocity dispersions than the OHDHS sample. The number of expected candidates with a $V$ velocity less than $-400 \mathrm{~km} \mathrm{~s}^{-1}$, compared with the null detection, implies that the fraction of dark matter halo made of such objects is less than $1 \%$ for a 12 Gyr halo, or that the halo is older than 12 Gyr.

In a more general way, this study shows that care must be taken when one tries to interpret the nature of high proper motion white dwarfs in photographic surveys (e.g. Knox et al. 1999; Ibata et al. 2000; Monet et al. 2000), as the thick disc population is a non-negligible part of high proper motion selected sample, and as thick disc white dwarfs cannot be easily distinguished from halo white dwarfs. As also shown by Crézé et al. (2001), the search for halo white dwarfs needs to be performed in deeper astrometric surveys, when the thick disc becomes non dominant over the expected halo white dwarfs population in high proper motion selected samples.

Acknowledgements. The authors thank G. Chabrier and L. Koopmans for fruitful discussions.

\section{References}

Bergeron, P., Wesemael, F., \& Beauchamp, A. 1995, PASP, 107, 1047

Bienaymé, O., Robin, A. C., \& Crézé, M. 1987a, A\&A, 180, 94 Bienaymé, O., Robin, A. C., \& Crézé, M. 1987b, A\&A, 186, 359

Chabrier, G. 1999, ApJ, 513, L103

Chabrier, G., Brassard, P., Fontaine, G., \& Saumon, D. 2000, ApJ, 543, 216

Crézé, M., Robin, A. C., Mohan, V., Cuillandre, J.-C., \& Reylé, C. 2001, A\&A, submitted

Gibson, B. K., \& Flynn, C. 2001, Science, preprint [astro-ph0104255]

Gould, A., Flynn, C., \& Bahcall, J. N. 1998, ApJ, 503, 798

Graff, D. S. 2001, Science, preprint [astro-ph0104210]

Hansen, B. M. S. 2001, ApJ, preprint [astro-ph0105018]

Haywood, M., Robin, A. C., \& Crézé, M. 1997, A\&A, 320, 440

Ibata, R., Irwin, M., Bienaymé, O., Scholz, R., \& Guibert, J. 2000, ApJ, 532, L41

Knox, R. A., Hawkins, M. R. S., \& Hambly, N. C. 1999, MNRAS, 306, 736

Koopmans, L. V. E., \& Blandford, R. D. 2001, MNRAS, preprint [astro-ph0107358]

Isern, J., Garcia-Berro, E., Hernanz, M., Mochkovitch, R., \& Torres, S. 1998, ApJ, 503, 239

Liebert, J., Dahn, C. C., Harris, H. C., \& Legget, S. K. 1999, ASP Conf. Ser., 169, 51

Monet, D. G., Fisher, M. D., Liebert, J., et al. 2000, AJ, 120, 1541

Ojha, D. K., Bienaymé, O., Robin, A. C., Crézé, M., \& Mohan, V. 1996, A\&A, 311, 456

Oppenheimer, B. R., Hambly, N. C., Digby, A. P., Hodgkin, S. T., \& Saumon, D. 2001, Science, 292, 698

Reylé, C., \& Robin, A. C. 2001, A\&A, 373, 886

Reid, I. N., Sahu, K. C., \& Hawley, S. L. 2001, ApJ, preprint [astro-ph0104110]

Robin, A. C., \& Chen, B. 1993, AIP Conf. Proc. 278, Back to the Galaxy, 384

Sion, E. M., \& Liebert, J. 1977, ApJ, 213, 468 